\title{
FMR1-dependent variability of ovarian aging patterns is already apparent in young oocyte donors
}

Norbert Gleicher ${ }^{1,2^{*}}$, Ann Kim¹, David H Barad ${ }^{1,2}$, Aya Shohat-Tal ${ }^{1}$, Emanuela Lazzaroni ${ }^{1}$, Tamar Michaeli', Ho-Joon Lee', Vitaly A Kushnir ${ }^{1}$ and Andrea Weghofer ${ }^{1,3}$

\begin{abstract}
Background: Hypothesizing that redundant functional ovarian reserve (FOR) at young ages may clinically obfuscate prematurely diminished FOR (PDFOR), we investigated in young oocyte donors genotypes and sub-genotypes of the FMR1 gene, in prior studies associated with specific ovarian aging patterns, and determined whether they already at such young age were associated with variations in ovarian reserve (OR). We also investigated racial as well as FMR1 associations with menarcheal age in these donors.

Methods: In a cohort study we investigated 157 oocyte donor candidates and, based on the $95 \% \mathrm{Cl}$ of $\mathrm{AMH}$, divided them into normal age-specific (AMH greater or equal to $2.1 \mathrm{ng} / \mathrm{mL} ; \mathrm{n}=121)$ and PDFOR $(\mathrm{AMH}<2.1 \mathrm{ng} / \mathrm{mL}$; $n=36$ ). We then assessed associations between numbers of trinucleotide repeat (CGGn) on the FMR1 gene and FOR (based on anti-Müllerian hormone, $\mathrm{AMH}$ ).

Results: FMR1 did not associate with AMH overall. Amongst 36 donors with PDFOR, 17 (42\%) presented with at least one low (CGGn $<26$ ) allele. Remaining donors with normal FOR presented with significantly more CGGn greater or equal to $26(73.6 \%$ vs. $26.4 \% ; P=0.024)$ and higher $\mathrm{AMH}(\mathrm{P}=0.012)$. This finding was mostly the consequence of interaction between FMR1 (CGGn $<26$ vs. CGGn greater or equal to 26) and race $(P=0.013)$, with Asians most responsible $(P=0.009)$. Menarcheal age was in donors with normal FOR neither associated with race nor with FMR1 status. In donors with PDFOR race was statistically associated with CGGn ( $P=0.018)$, an association primarily based on significantly delayed age of menarche in African donors with CGGn $<26$ in comparison to African donors with CGGn greater or equal to $26(P=0.019)$, and Caucasian $(P=0.017)$ and Asian donors $(P=0.025)$ with CGGn $<26$.
\end{abstract}

Conclusions: CGGn on FMR1 already at young ages affects FOR, but is clinically apparent only in cases of PDFOR. Screening for low FMR1 CGGn $<26$ at young age, thus, appears predictive of later PDFOR.

Keywords: Fragile X gene, FMR1 gene, Ovarian reserve, Anti-müllerian hormone (AMH), Oocyte donor

\section{Background}

The FMR1 gene, located on the $\mathrm{X}$ chromosome, is widely tested in prenatal medicine in attempts to prevent the so-called fragile $\mathrm{X}$ syndrome, representing the onegenerational expansion of a premutation-range $\mathrm{CGG}_{\mathrm{n}=55-200}$ to a full mutation, characterized by a $\mathrm{CGG}_{\mathrm{n}>200}$. Based on a newly defined normal range of $\mathrm{CGG}_{\mathrm{n}=26-34}$, it has only

\footnotetext{
* Correspondence: ngleicher@the chr.com

${ }^{1}$ Center for Human Reproduction, 10021, New York, NY, USA

${ }^{2}$ Foundation for Reproductive Medicine, 10021, New York, NY, USA

Full list of author information is available at the end of the article
}

recently in a number of publications been associated with ovarian aging. Different mutations of the gene appear associated with different rates of follicle depletion.

Lledo et al. recently, however, suggested that FMR1 screening should not be considered in pre-assessing potential responses to ovarian stimulation [1], implying that different FMR1 mutations do not correlate with variations in functional ovarian reserve (FOR). We, in contrast, previously reported specific ovarian FMR1 genotypes and sub-genotypes, defined by normal range of $\mathrm{CGG}_{\mathrm{n}=26-34}$ (median, $\mathrm{CGG}_{\mathrm{n}=30}$ ), associated with distinct ovarian aging

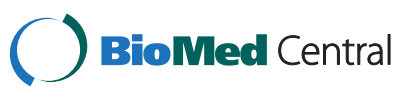


patterns [2,3]. These varying mutations of the FMR1 gene have since also proven associated with risk towards autoimmunity [4] and, potentially, BRCA1/BRCA2-associated cancer risks [5]. Moreover, Hoffman et al. recently reported in a mouse analogue of human premutation range $\left(\mathrm{CGG}_{\mathrm{n}=55-200}\right)$ primary ovarian insufficiency (POI) that the model, in comparison to wild type mice, demonstrated clear anatomic evidence of premature ovarian aging (POA), including accelerated follicle loss [6].

Lledo and associates reached their conclusions, investigating oocyte yields in young oocyte donors [1]. Like them, we previously also had been unable to demonstrate differences in FOR in young oocyte donors [2]. We, therefore, to a degree based on their publication, hypothesized that young oocyte donors, likely, do not represent suitable subjects for such studies, as, at such young ages, even impeded FOR may still create enough redundancy to produce excellent oocyte yields [4].

Our earlier study involved, however, only 34 young women [2]. We, therefore, decided to revisit the question based on a larger patient sample, and a more sophisticated study protocol. We here, therefore, investigated a much larger egg donor population, stratified for normal FOR and PDFOR.

\section{Methods}

This study investigated 157 consecutive oocyte donors, selected for our center's oocyte donor pool. We investigated in this patient cohort whether their AMH levels at time of initial presentation varied in association with different FMR1 mutations, adjusted for race. We, in addition, also investigated whether the donors' menarcheal age was affected by either race and/or FMR1 status.

Less than five percent of applicants are accepted into our center's oocyte donor pool. Donor selection involves an initial screening step by questionnaire, followed by two rounds of face-to-face interview and a final medical testing round. Once candidates have passed this final testing round, they become eligible for matching with recipients. Once selected by a recipient, the donor undergoes a second testing round in accordance with guidelines issued by the United States Food and Drug Administration, the federal agency overseeing gamete donations in the United States. Only if this testing round is satisfactory is the donor considered "matched".

In this study AMH and FMR1 testing was performed on the day of the donor's second face-to-face interview at the center. All patients at our center undergo routine FMR1 testing to screen for one-generational expansion risk in offspring towards the fragile $\mathrm{X}$ syndrome. The test is neither performed nor used to predict reproductive performance of potential donors. Donor selection is, therefore, not influenced by what genotypes or subgenotypes of FMR1 a given donor candidate represents.
Our center's Institutional Review Board (IRB), however, based on prior published FMR1 studies from our center, requires that donor candidates be informed if their FMR1 mutations are believed to potentially denote risk towards PDFOR.

Ovarian FMR1 genotypes and sub-genotypes, as previously described, are based on a normal $\mathrm{CGG}_{\mathrm{n}=26-34}$ range $[2,3]$. This means that patients are considered to have normal (norm) genotypes if both alleles are in normal range. They are considered to be heterozygous (het) if one allele is outside, and homozygous (hom) if both alleles are outside of normal range. Depending on whether abnormal alleles are above or below normal range, het and hom genotypes are further sub-divided into sub-genotypes. Het genotypes are sub-divided into het-norm/high and hetnorm/low and hom genotypes into hom-high/high, homhigh/low and hom-low/low. Every allele of $\mathrm{CGG}_{\mathrm{n}<26}$ is, therefore, considered a low allele. Norm and high alleles in this manuscript are combined as $\mathrm{CGG}_{\mathrm{n} \geq 26}$. FMR1 and $\mathrm{AMH}$ assays were performed by routine commercial assays, as previously reported $[2,3]$.

At time of acceptance into our center's donor pool, the mean age of donors was $24.5 \pm 3.2$ years, their body mass index (BMI) was $21.3 \pm 2.4 \mathrm{~kg} / \mathrm{m}^{2}$ and their mean $\mathrm{AMH}$ was $4.2 \pm 2.6 \mathrm{ng} / \mathrm{mL}$ (Table 1 ).

The lower $95 \% \mathrm{CI}$ for all AMH values, at $<2.1 \mathrm{ng} / \mathrm{mL}$, was defined as diagnostic for PDFOR. Donors with AMH above this range were, therefore, considered to have normal FOR.

Because distribution patterns of FMR1 genotypes and sub-genotypes vary between races $[7,8]$, all data were adjusted for race. Racial assignments were made according

Table 1 Baseline characteristics of all oocyte donors

\begin{tabular}{lc}
\hline Number of donor candidates & $\mathbf{1 5 7}$ \\
\hline Caucasian (\%) & $109(69.4)$ \\
Asian (\%) & $26(16.6)$ \\
African (\%) & $22(14.0)$ \\
Age at presentation (years) & $24.6 \pm 3.2$ \\
Age at menarche (years) & $12.9 \pm 1.5$ \\
BMI (kg/m2) & $21.3 \pm 2.4$ \\
AMH (ng/mL) & $4.2 \pm 2.6$ \\
FMR1 [n (\%)]: norm & $83(52.9)$ \\
het & $55(35.0)$ \\
het-norm/high & $16(10.2)$ \\
het-norm/low & $39(24.8)$ \\
hom & $19(12.1)$ \\
hom-high/high & $9(5.7)$ \\
hom-high/low & $5(3.2)$ \\
hom-low/low & $5(3.2)$ \\
\hline
\end{tabular}


to National Institutes of Health Criteria, with Hispanic donors assigned according to their racial self-definition.

Differences between continuous and categorical data were calculated using Kruskal-Wallis, Mann-Whitney U, two-way analysis of variance and Chi-Square tests, with $\mathrm{P}<0.05$ considered statistically significant. All post hoc procedures were performed using the Sidak test. Statistical analyses were undertaken using the Statistical Package for the Social Sciences 17.0 (SPSS, Chicago, IL, USA).

Oocyte donor candidates, like all other patients at our center, at time of initial consultation sign an informed consent, which allows for use of their medical record for research purposes, as long as the patient's identity is protected and the medical record remains confidential. Both conditions were met for this study, and the study, therefore, qualified for expedited review by the center's IRB. Like infertility patients, oocyte donor candidates also sign specific informed consents for genetic testing, including FMR1 testing. Though, as already noted before, $F M R 1$ test results in our program are not utilized to select oocyte donors, our IRB mandated that donors be informed about the potential clinical significance if they demonstrated other than norm genotypes.

\section{Results}

Table 1 summarizes the characteristics of all 157 oocyte donor candidates. As the table demonstrates over twothirds were Caucasian; the remaining almost evenly split between women of Asian and African descent.

The FMR1 genotype distribution was 83 norm (52.9\%), 55 het (35.0\%) and 19 hom (12.1\%). Het were further sub-divided into 16 het-norm/high (10.2\%) and 39 hetnorm/low (24.8\%), while hom was sub-divided into 9 hom-high/high (5.7\%), 5 hom-high/low (3.2\%) and 5 homlow/low (3.2\%). A total of 49 (31.2\%) donors, therefore, demonstrated at least one low allele of $\mathrm{CGG}_{\mathrm{n}<26}$, while 108 donors presented exclusively with $\mathrm{CGG}_{\mathrm{n} \geq 26}$, representing norm $\left(\mathrm{CGG}_{\mathrm{n}=26-34}\right)$ or high $\left(\mathrm{CGG}_{\mathrm{n}>34}\right)$ alleles.

Figures 1A, B present FOR data, based on AMH measurements, with A representing donors with normal FOR and $\mathrm{B}$ representing donors with PDFOR. Differences are obvious, with normal FOR donors demonstrating uniformly mean AMH levels above $4 \mathrm{ng} / \mathrm{mL}$, while donors with PDFOR uniformly demonstrate mean AMH below $2 \mathrm{ng} / \mathrm{mL}$.

When the 36 donors with PDFOR $(\mathrm{AMH}<2.1 \mathrm{ng} / \mathrm{mL})$ were investigated separately, differences started coming into focus: Among 36 donors with PDFOR, 17 (47.2\%) presented with at least one low FMR1 allele $\left(\mathrm{CGG}_{\mathrm{n}<26}\right)$ and $19(52.8 \%)$ with none. The remaining 121 donors with normal FOR $(A M H \geq 2.1 \mathrm{ng} / \mathrm{mL})$ were significantly more likely to have a $\mathrm{CGG}_{\mathrm{n} \geq 26}$ allele ( $73.6 \%$ vs. $26.4 \%$; $\left.\mathrm{P}=0.024\right)$.

Normal FOR donors with a low $\left(\mathrm{CGG}_{\mathrm{n}<26}\right)$ allele still demonstrated higher mean AMH levels than donors with
$\mathrm{CGG}_{\mathrm{n} \geq 26}(\mathrm{P}=0.012)$. As Figure 1A demonstrates, amongst Caucasian and African donors AMH levels were similar and did not differ significantly based on $\mathrm{CGG}_{\mathrm{n}}$. Asian donors, however, demonstrated, in comparison, significantly higher $\mathrm{AMH}$ in association with presence of at least one low $\left(\mathrm{CGG}_{\mathrm{n}<26}\right)$ allele $(\mathrm{P}=0.009)$. They, therefore, are likely primarily responsible for above described statistical association with $\mathrm{CGG}_{\mathrm{n}}$ for the whole group of donors with PDFOR.

These findings need to be contrasted to AMH levels in donors with PDFOR, where, independent of race, all donors presented with much lower AMH overall, but with higher AMH levels in absence of a low allele (Figure 1B) in each race, establishing an almost significant trend $(P=0.057)$ in reversal to observation in donors with normal FOR. Figure 1B further demonstrates that Caucasians and African donors once again demonstrated similar $\mathrm{AMH}$ levels, while Asian donors, again, demonstrated highest AMH levels, here, however, independent of $\mathrm{CGG}_{\mathrm{n}}$.

When investigating menarcheal age, neither race nor FMR1 status mattered in donors with normal FOR (Figure 1C). Donors with PDFOR, however, demonstrated significant differences by demonstrating significant interaction between FMR1 $\mathrm{CGG}_{\mathrm{n}}$ and race $(\mathrm{P}=0.018)$, primarily based on significantly later menarche in African women with low $\mathrm{CGG}_{\mathrm{n}<26}$. African donors with low $\mathrm{CGG}_{<26}$ demonstrated significantly later menarche than African donors with $C G_{n \geq 26}(P=0.019)$, and than low FMR1 Caucasian $(\mathrm{P}=0.017)$ and Asian $(\mathrm{P}=0.025)$ donors (Figure 1D).

\section{Discussion}

A so-called premutation-range genotype $\left(\mathrm{CGG}_{\mathrm{n}=55-200}\right)$ of the FMR1 gene has for decades been associated with highly increased risk towards primary ovarian insufficiency (POI), also called premature ovarian failure (POF) $[6,9]$. Specific ovarian functions of the FMR1 gene have, however, only in recent years been described in association with newly described FMR1 mutations, which were established based on a normal range of $\mathrm{CGG}_{\mathrm{n}=26-34}[2,3]$.

We started to suspect a specific ovarian function of FMR1 after noting a large distribution peak at $\mathrm{CGG}_{\mathrm{n}=31-32}$ in a quite dated publication by $\mathrm{Fu}$ and associates [10], suggesting a potentially new normal $\mathrm{CGG}_{\mathrm{n}}$ distribution range for a new function of the gene. In assessing $C G_{n}$ in various populations, we then demonstrated that, independent of racial background, a normal "ovarian" CGG triple nucleotide range, indeed, appeared at $\mathrm{CGG}_{\mathrm{n}=26-34 \text {, }}$ close to, and including the distribution peak reported by $\mathrm{Fu}$ et al. [2,11]. Defining such a normal range we then, based on $\mathrm{CGG}_{\mathrm{n}}$, can classify her as norm, het and hom [2]. Het and hom genotypes can be further sub-divided, based on whether abnormal alleles have $\mathrm{CGG}_{\mathrm{n}}$ above (high) or below (low) normal range [3]. 


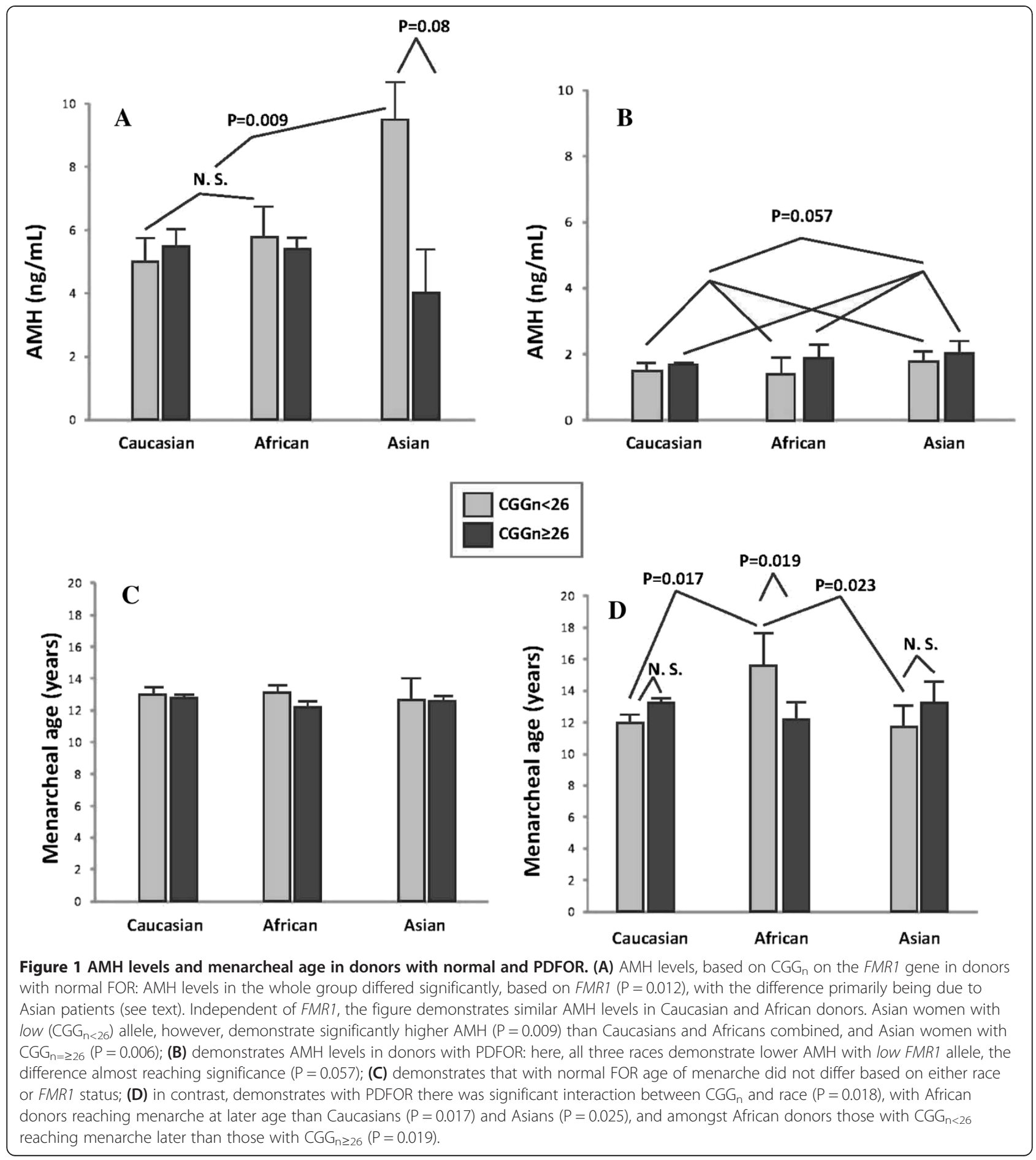

FMR1 genotypes and sub-genotypes have since been associated with different ovarian aging patterns [2,3], different pregnancy rates in association with in vitro fertilization (IVF) [3] but also with risk towards autoimmunity [3,8] and, likely, BRCA1/2 mutation-associated cancer risks [5], suggesting, yet additional, previously unknown biological functions for the FMR1 gene.
The most interesting evolving mutation of $F M R 1$, so far, has been the het-norm/low sub-genotype, characterized by one allele in norm range and one at $\mathrm{CGG}_{\mathrm{n}<26}$. This low sub-genotype has been associated with a polycystic ovary-like ovarian phenotype, which, because of rapid recruitment, and therefore depletion of FOR, quickly deteriorates into a phenotype characterized by premature 
diminished FOR [3]. It is also the genotype associated with lowest IVF pregnancy rates [3], autoimmunity [3,5] and, likely, $B R C A 1 / 2$-associated cancer risks [5]. Interestingly, its "sibling" sub-genotype, het-norm/high, is associated with highest FOR at very advanced female ages [12], and is practically protective of autoimmune [3] and BRCA1/2associated cancer risks [5].

Here presented study for the first time offered the opportunity to examine the effects of the FMR1 gene on the ovarian aging process in a very young, and apparently normal cohort of females: young egg donors. For the complete study cohort, $\mathrm{CGG}_{\mathrm{n}}$ of the FMR1 gene did not affect OR. FMR1 effects, however, did become apparent in donors who already at such young age (mean 24.6 years) suffered from PDFOR.

The study included 36 such donors, 17 (47.2\%) with a low allele and 19 (52.8\%) without. Donors with PDFOR were significantly less likely to present with $\mathrm{CGG}_{\mathrm{n} \geq 26}$ than donors with normal FOR $(\mathrm{P}=0.024)$. In young women with absolutely normal FOR, redundancy of extra ovarian reserve, therefore, indeed, appears substantial enough to prevent FMR1-driven differences, caused by low FMR1 mutations, to become clinically apparent. We suspected so much in a response to the recent publication by Lledo et al. [1].

Higher AMH values, as observed in African and Asian donors with normal FOR and low FMR1 $\mathrm{CGG}_{\mathrm{n}<26}$ than in donors with $C_{G G} G_{n \geq 26}(P=0.012$, Figure $1 \mathrm{~A})$, may at first glance surprise since higher $\mathrm{AMH}$ denotes better FOR, resulting in better quantitative and qualitative IVF outcomes [13]. Since these high AMH values appear primarily the consequence of observations in Asian women (Figure 1A), they, however, have to be viewed with caution because young Asian women are not typically known to present with high FOR $(7,8)$. Moreover, though low FMR1 mutations of the het-norm/low sub-genotype have been associated with the poorest IVF outcomes amongst all FMR1 genotypes [3], this observation, too, has to be understood within a proper context.

The het-norm/low sub-genotype of the FMR1 gene is at young ages characterized by a PCOS-like ovarian phenotype. PCOS, in turn, is associated with high FOR and, therefore, high AMH levels. Young women with low FMR1 mutations, while still during the PCOS-like phase of their ovarian development, therefore, are expected to demonstrate high AMH values. Considering small numbers of Asian and African donors in this study, here observed high AMH levels, especially amongst Asian donors, have to be viewed with caution. Reported studies actually suggest a preponderance of low FMR1 alleles in African rather than Asian women $[7,8]$.

This PCOS-like ovarian phenotype in low FMR1 mutation carriers is, because of relatively rapid recruitment, also characterized by early follicle depletion, by mid-age transforming many of these women into patients with PDFOR [3]. Young oocyte donors with low FMR1 alleles and high FOR, therefore, may reflect ovarian phenotypes pre-depletion.

This interpretation of here reported data is also supported by the previously noted distribution of FMR1 mutations amongst donors: Those who already had entered PDFOR $(\mathrm{n}=36)$, in almost half of cases $(17 / 36$; 47.2\%) demonstrated low FMR1 alleles, while those with normal FOR exhibited $\mathrm{CGG}_{\mathrm{n}<26}$ in only $32 / 121$ (26.5\%; $\mathrm{P}=0.018$ ).

Inversion of $\mathrm{AMH}$ data, as demonstrated in Figures $1 \mathrm{~A}$ (normal FOR; overall higher AMH with $\mathrm{CGG}_{\mathrm{n}<26}$ ) and 1B (PDFOR; higher AMH with $\mathrm{CGG}_{\mathrm{n} \geq 26}$ ), therefore, may or may not be race-dependent. Data, however, do appear to suggest that the FMR1 gene, indeed, does affect FOR already at the young ages, though such effects are in many young women not yet detectable by $\mathrm{AMH}$ assessments at such early stages. Here presented data, therefore, are in agreement with previously published FMR1 data in older infertile patients, which suggest that a low allele is prognostically predictive of PRFOR and poorer pregnancy chances [3].

These data, however, in addition for the first time in exclusively young, and apparently healthy females demonstrate that a low FMR1 $\mathrm{CGG}_{\mathrm{n}<26}$ allele appears predictive of PDFOR. While earlier cross-sectional studies from our center in infertile patient populations were suggestive of such an interpretation, here presented data offer convincing evidence in a very young, and seemingly fertile, highly selected egg donor population.

Screening for low FMR1 mutations in young women, therefore, may offer the opportunity to detect genetically predisposed women towards PDFOR already at very young ages. Confirmation of risk by detection of a low FMR1 allele then would allow for longitudinal FOR assessments with $\mathrm{AMH}$ and FSH to either confirm or refute a diagnosis of PDFOR.

This study also demonstrates that, even in the early 20 s, some young women with low FMR1 mutations, and therefore at risk for PDFOR, still have so much overabundant FOR that AMH measurements will not yet allow for a diagnosis. Such women, therefore, will have to be followed longitudinally with serial AMH and FSH measurements. Confirmation of PDFOR at much younger age than when currently PDFOR diagnoses are reached, will allow affected women to plan their reproductive life accordingly and/or consider fertility preservation through social oocyte cryopreservation with much better success and at lower cost than with later diagnosis.

The importance of low FMR1 mutations for the ovarian aging process is further emphasized by here reported menarcheal age data. While the total donor population again does not reveal any race- or FMR1-based differences 
(Figure 1C), donors with PDFOR do demonstrate significant differences based on race and FMR1 mutation (Figure 1D). Here, significant interaction between $\mathrm{CGG}_{\mathrm{n}}$ and race became apparent $(\mathrm{P}=0.018)$, with African donors entering menarche at older age than either Caucasians or Asians.

That African donors behave so differently from Asian and Caucasian donors has, once again, to be viewed with caution and considered preliminary, in view of the relatively small size of the African donor pool in this study. Studying much older infertile women, we recently reported that, adjusted for age and race, diminished functional ovarian reserve (DFOR) in infertile women was statistically highly associated with younger age at menarche [13]. Since among all genotypes and sub-genotypes low FMR1 mutations appear most closely associated with DFOR, one would expect to find younger menarcheal age amongst donors with $\mathrm{CGG}_{\mathrm{n}<26}$ than amongst donor with $\mathrm{CGG}_{\mathrm{n} \geq 26}$. That Asians demonstrate such a trend and Caucasians to a lesser extent but that Africans demonstrate to a significant degree exactly the opposite (Figure 1D) may suggest racial differences as to how the $F M R 1$ gene affects ovarian aging. This study, however, does not allow for further comments on the subject.

This study's major weakness is the small size $(\mathrm{n}=36)$ of donors with PDFOR, as they ended up representing the primary study population, demonstrating significant positive associations. Considering the small size of this cohort, the robustness of here reported statistical findings is actually rather surprising. Our findings, however, do require confirmation in larger young patient populations with PDFOR before here proposed conclusions can be considered confirmed.

Maybe the most surprising finding of this study was, however, the undisputed observation that 36/157 (22.9\%) of carefully selected young oocyte donors, based on $95 \% \mathrm{CI}$ for $\mathrm{AMH}$, were found with great likelihood to suffer from PDFOR. This observation demonstrates the limitations of currently used oocyte donor selection methods. As noted under Materials and Methods, our center, based on instructions from our center's IRB, currently does not utilize FMR1 genotypes and sub-genotypes in egg donor selection. Here presented data suggest that FMR1-testing may, however, indeed, have a place in the egg donor selection process.

Finally, this study, once again, points to the importance of racial differences in reproductive medicine. We on repeated occasions have made this point in recent publications $[4,11]$, stressing the importance of racial adjustments in studies of reproductive outcomes.

\section{Conclusions}

CGGn on FMR1 already at young ages affects FOR, but is clinically apparent only in cases of PDFOR. Screening for low FMR1 $\mathrm{CGG}_{\mathrm{n}<26}$ at young age, thus, appears predictive of later PDFOR.

\section{Competing interests}

N.G, A.W. and D.H.B. have in the past received research support, speakers' honoraria and travel funds from various pharmaceutical and medical device companies, none, however, related to the subject of this paper. N.G. and D.H. B. are listed as co-inventors of two awarded U.S. patents, claiming therapeutic benefits for DHEA, and potentially other androgens, in women with DOR. Both authors have other pending patent applications, regarding DHEA, and other androgens, and the FMR1 gene's effects on ovaries. N.G. owns shares in Fertility Nutraceuticals, LLC, a company that offers a DHEA product. N.G. and D.H.B. are receiving patent royalties from this company. N.G. is also the owner of The $\mathrm{CHR}$, where this research was conducted. Other authors have no competing interests to declare.

\section{Authors' contributions}

NG and DHB contributed equally to the manuscript. NG contributed to study design and data analysis, and wrote the manuscript, AK and DHB performed data analyses and statistical analyses, AW contributed to study design. All authors read and approved the final manuscript.

\section{Acknowledgments}

The study was supported by the Foundation for Reproductive Medicine and intramural grants from the Center for Human Reproduction.

\section{Author details}

${ }^{1}$ Center for Human Reproduction, 10021, New York, NY, USA. ${ }^{2}$ Foundation for Reproductive Medicine, 10021, New York, NY, USA. ${ }^{3}$ Department of Gynecologic Endocrinology and Reproductive Medicine, Medical University Vienna, 1090, Vienna, Austria.

Received: 6 June 2013 Accepted: 8 August 2013

Published: 16 August 2013

\section{References}

1. Lledo B, Guerrero J, Ortiz JA, Morales R, Ten J, Llacer J, Gimenez J, Bernabeu R: Intermediate and normal sized CGG repeats on the FMR1 gene does not negatively affect donor ovarian response. Hum Reprod 2012, 27:609-614.

2. Gleicher N, Weghofer A, Barad DH: Ovarian reserve determinations suggest new function of FMR1 (fragile $\mathrm{X}$ gene) in regulating ovarian ageing. Reprod Biomed Online 2010, 20:768-755.

3. Gleicher N, Weghofer A, Lee IH, Barad DH: FMR1 genotype with autoimmune-associated polycystic ovary-like phenotype and decreased pregnancy chances. PLoS One 2010, 5:315303.

4. Gleicher N, Weghofer A, Barad DH: Intermediate and normal sized CGG repeats on the FMR1 gene does not negatively affect donor ovarian response. Hum Reprod 2012, 27(7):2241-2241. author reply 2242-2243.

5. Weghofer A, Tea M-K, Barad DH, Kim A, Singer CF, Wagner K, Gleicher N: RCA1/2 mutations appear embryo lethal in humans, unless rescued by low $(\mathrm{GCCn}<26)$ FMR1 sub-genotypes. PLoS One 2012. in press.

6. Hoffman G, Le WW, Entezam A, Otsuka N, Tong Z-B, Nelson L, Flaws JA, McDonald JH, Jafar S, Usdin K: Ovarian abnormalities in a mouse model of Fragile X Primary Insufficiency. J Histochem Cytochem 2012, 60:439.

7. Gleicher N, Kim A, Weghofer A, Barad DH: Differences in ovarian aging patterns between races are likely related to ovarian genotypes and sub-genotypes of the FMR1 gene. Reprod Biol Endocrinol 2012, 10:77.

8. Gleicher N, Weghofer A, Lee IH, Barad DH: Association of FMR1 genotypes with in vitro fertilization (IVF) outcomes based on ethnicity/race. PLoS One 2011, 6:e18781.

9. Wittenberger MD, Hagerman RJ, Sherman SL, McConkie-Rosell A, Welt CK, Rebar RW, Corrigan EC, Simpson JL, Nelson LM: The FMR1 premutation and reproduction. Fertil Steril 2007, 87:456-465.

10. Fu YJ, Kuhl DP, Pizzuti A, Pieretti M, Sutcliffe JS, Richards S, Verkerk AJ, Holden JJ, Fenwick RG Jr, Warren ST, et al: Variation of the CGG repeat at the fragile $\mathrm{X}$ site results in genetic instability: resolution of the Sherman paradox. Cell 1991, 20(67):1074-1058.

11. Gleicher N, Weghofer A, Oktay K, Barad DH: Effects of race/ethnicity on triple CGG counts on the FMR1 (fragile X) gene in infertile women. Reprod Biomed Online 2010, 20:485-491. 
12. Gleicher N, Weghofer A, Kim A, Barad DH: The impact in older women of ovarian FMR1 genotypes and sub-genotypes on ovarian reserve. PLoS One 2012, 7:e33638.

13. Weghofer A, Kim A, Barad DH, Gleicher N: Age at menarche: a predictor of diminished ovarian function? Fertil Steril 2013. doi:10.1016/j. fertnstert.2013.05.042. [Epub ahead of print]

doi:10.1186/1477-7827-11-80

Cite this article as: Gleicher et al:: FMR1-dependent variability of ovarian aging patterns is already apparent in young oocyte donors. Reproductive Biology and Endocrinology 2013 11:80.

\section{Submit your next manuscript to BioMed Central and take full advantage of:}

- Convenient online submission

- Thorough peer review

- No space constraints or color figure charges

- Immediate publication on acceptance

- Inclusion in PubMed, CAS, Scopus and Google Scholar

- Research which is freely available for redistribution 